\title{
A Two-parameter Rama Distribution
}

\section{U. Umeh Edith, T. Umeokeke Ebele* and A. Ibenegbu Henrietta \\ Department of Statistics, Nnamdi Azikiwe University, Awka, Nigeria \\ e-mail: eu.umeh@unizik.edu.ng; editus2002@yahoo.com; amuche4mary@yahoo.com \\ *Corresponding author's e-mail: umeokekeebele@gmail.com}

\begin{abstract}
In this paper, a two-parameter Rama distribution is proposed. This is coined from Lindley distribution and Rama distribution. Its mathematical and statistical properties which include its shapes, moment, coefficient of variation, skewness, kurtosis, index of dispersion, hazard rate function, mean residual life function, stochastic ordering, mean deviation; Bonferroni and Lorenz curves are also discussed. The estimation of parameters has been X-rayed using methods of moment and maximum likelihood. Also AIC and BIC are used to test for the goodness of fit of the model which is applied to a real life data of hepatitis B patients. This new distribution is compared with Rama, 2-parameter Akash, 2-parameter Lindley, Akash, Shanker, Ishita, Lindley and Exponential distributions in order to determine the efficiency of the new model.
\end{abstract}

\section{Introduction}

Shanker [1] proposed a continuous lifetime distribution, known as Rama distribution defined by its probability density function (pdf) and cumulative distribution function (cdf) given below

$$
f(x ; \theta)=\frac{\theta^{4}}{\theta^{3}+6}\left(1+x^{3}\right) e^{-\theta x} ; \quad x>0, \theta>0,
$$

Received: June 2, 2019; Accepted: July 30, 2019

2010 Mathematics Subject Classification: 97K80, 62Nxx.

Keywords and phrases: Rama distribution, mathematical and statistical properties, estimation of parameters, goodness of fit.

Copyright (C) 2019 T. Umeokeke Ebele et al. This is an open access article distributed under the Creative Commons Attribution License, which permits unrestricted use, distribution, and reproduction in any medium, provided the original work is properly cited. 


$$
F(x ; \theta)=1-\left[1+\frac{\theta^{3} x^{3}+3 \theta^{2} x^{2}+6 \theta x}{\theta^{3}+6}\right] e^{-\theta x} ; \quad x>0, \theta>0 .
$$

Rama distribution is a convex combination of exponential distribution $(\theta)$ and gamma distribution $(4, \theta)$ with their mixing proportions $\frac{\theta^{3}}{\theta^{3}+6}$.

Shanker [1] has discussed its various mathematical properties and shows that (1.1) has a better model for modeling lifetime data from medical science and engineering than that of Lindley [2], Shanker [3], Shanker [4], Shanker [5], Shanker [6] and exponential distributions. The first four moments about origin of Rama distribution obtained by Shanker [1] are given by

$$
\begin{array}{ll}
\mu_{1}^{\prime}=\frac{\theta^{3}+24}{\theta\left(\theta^{3}+6\right)}, & \mu_{2}^{\prime}=\frac{2\left(\theta^{3}+60\right)}{\theta^{2}\left(\theta^{3}+6\right)}, \\
\mu_{3}^{\prime}=\frac{6\left(\theta^{3}+120\right)}{\theta^{3}\left(\theta^{3}+6\right)}, & \mu_{4}^{\prime}=\frac{24\left(\theta^{3}+210\right)}{\theta^{4}\left(\theta^{3}+6\right)} .
\end{array}
$$

Shanker [1] has discussed its important statistical properties including shapes of density function for varying values of parameters, hazard rate function; mean residual life function, stochastic ordering, mean deviations, Bonferroni and Lorenz curves and stressstrength reliability. He has also discussed the maximum likelihood estimation of the parameter and showed the application of Rama distribution.

The pdf and the cdf of Lindley distribution introduced by Lindley [2] are defined as

$$
\begin{gathered}
f(x ; \theta)=\frac{\theta^{2}}{\theta+1}(1+x) e^{-\theta x}, \\
F(x ; \theta)=1-\left[1+\frac{\theta x}{\theta+1}\right] e^{-\theta x} .
\end{gathered}
$$

Lindley distribution has been studied in detail by Ghitany et al. [7], its mathematical properties and statistical properties were discussed. Shanker and Shukla [8] have discussed two-parameter Akash distribution and its application. Shanker [5] has detailed and critical study on applications of exponential and Lindley distribution for modeling real lifetime data from biomedical sciences and engineering and observed that both 
exponential and Lindley are competing each other. Shanker [6] has discussed applications of Gamma and Weibull distributions for real lifetime data from engineering and biological sciences. Furthermore Shanker [1] has discussed the comparative study on lifetime data using Rama distribution and other distributions (Akash, Shanker, Aradhana, Amarendra, Sujatha, Lindley and Exponential) and showed that the Rama distribution is a best fit compared to others.

In this paper, a two-parameter Rama distribution (TPRD) which includes oneparameter Rama distribution of Shanker [1] as a particular case has been suggested. Its shapes, moments and moments based properties have been derived and discussed. The hazard rate function, mean residual life function, stochastic ordering, mean deviations, order statistics, Bonferroni and Lorenz curves of TPRD have been derived and discussed using method of moment and method of maximum likelihood. The goodness of fit of the proposed distribution has been discussed with a real life numerical data set and the fit has been compared with some well known lifetime distributions.

\section{A Two-parameter Rama Distribution}

A two-parameter Rama distribution (TPRD) with parameters $\theta$ and $\alpha$ is defined by its pdf and cdf

$$
f(x ; \theta, \alpha)=\frac{\theta^{4}}{\alpha \theta^{3}+6}\left(\alpha+x^{3}\right) e^{-\theta x} ; \quad x>0, \theta>0, \alpha \geq 0
$$

where $\theta$ is a scale parameter and $\alpha$ is a shape parameter. It reduces to Rama distribution (1.1) for $\alpha=1$. The p.d.f (2.1) can be shown as a mixture of exponential $(\theta)$ and gamma $(4, \theta)$ distributions as follows:

$$
f(x ; \theta, \alpha)=p h_{1}(x)+(1-p) h_{2}(x)
$$

where $p=\frac{\alpha \theta^{3}}{\alpha \theta^{3}+6}, h_{1}(x)=\theta e^{-\theta x}$ and $h_{2}(x)=\frac{\theta^{4} x^{3} e^{-\theta x}}{6}$.

The corresponding cumulative distribution function (cdf) of (2.1) can be expressed as

$$
F(x)=1-\left[1+\frac{\theta x\left(\theta^{2} x^{2}+3 \theta x+6\right)}{\alpha \theta^{3}+6}\right] e^{-\theta x} ; \quad x>0, \theta>0, \alpha \geq 0 .
$$


Thus, the graphs of the pdf and cdf are given below.
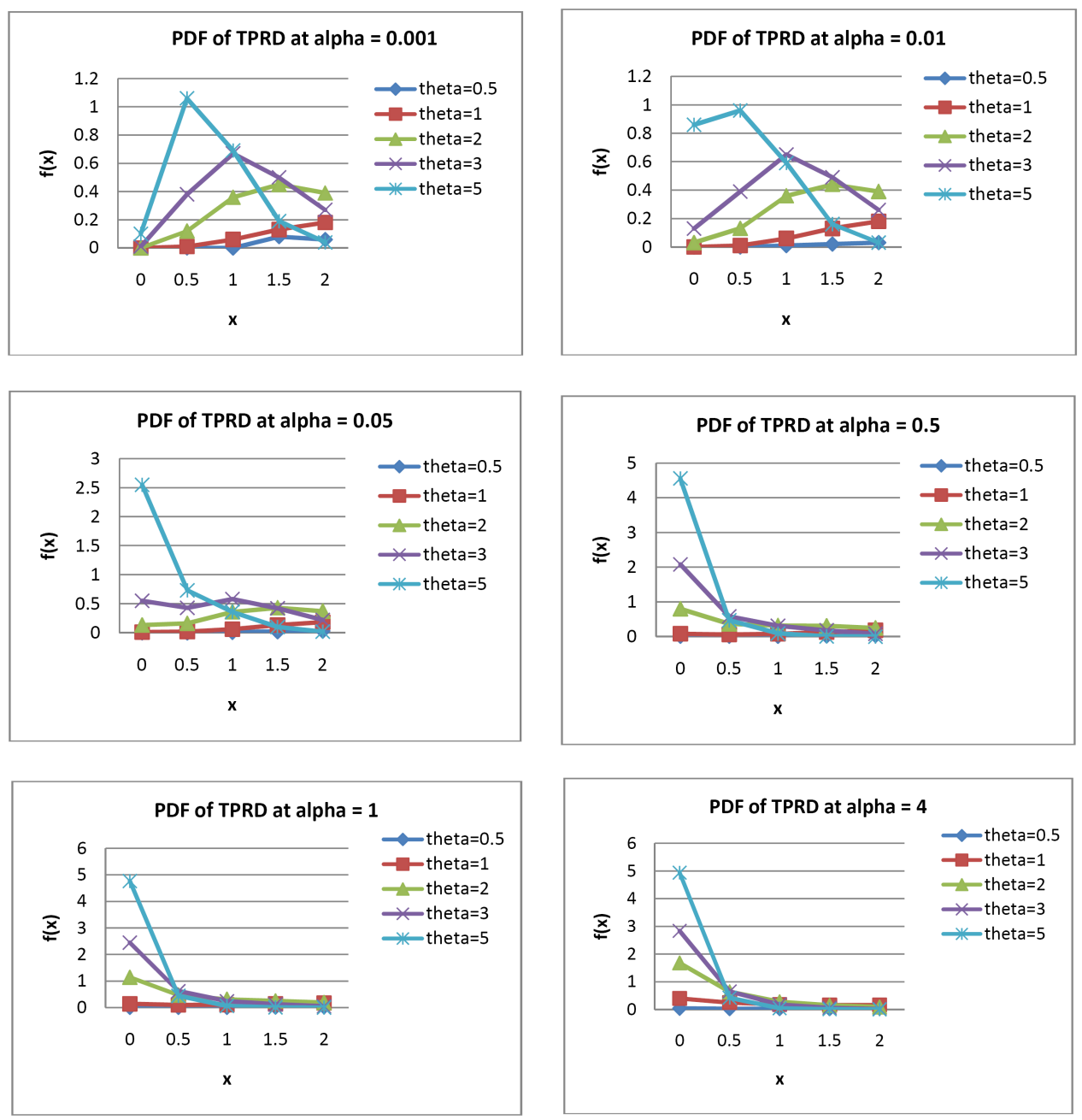

Figure 1. Behaviour of the PDF of TPRD for varying values of the parameters $\theta$ and $\alpha$.
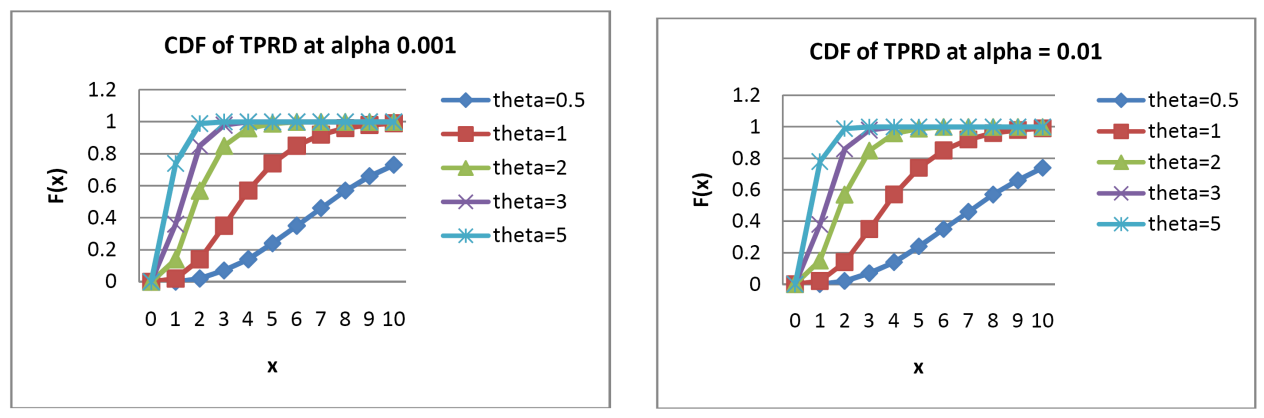

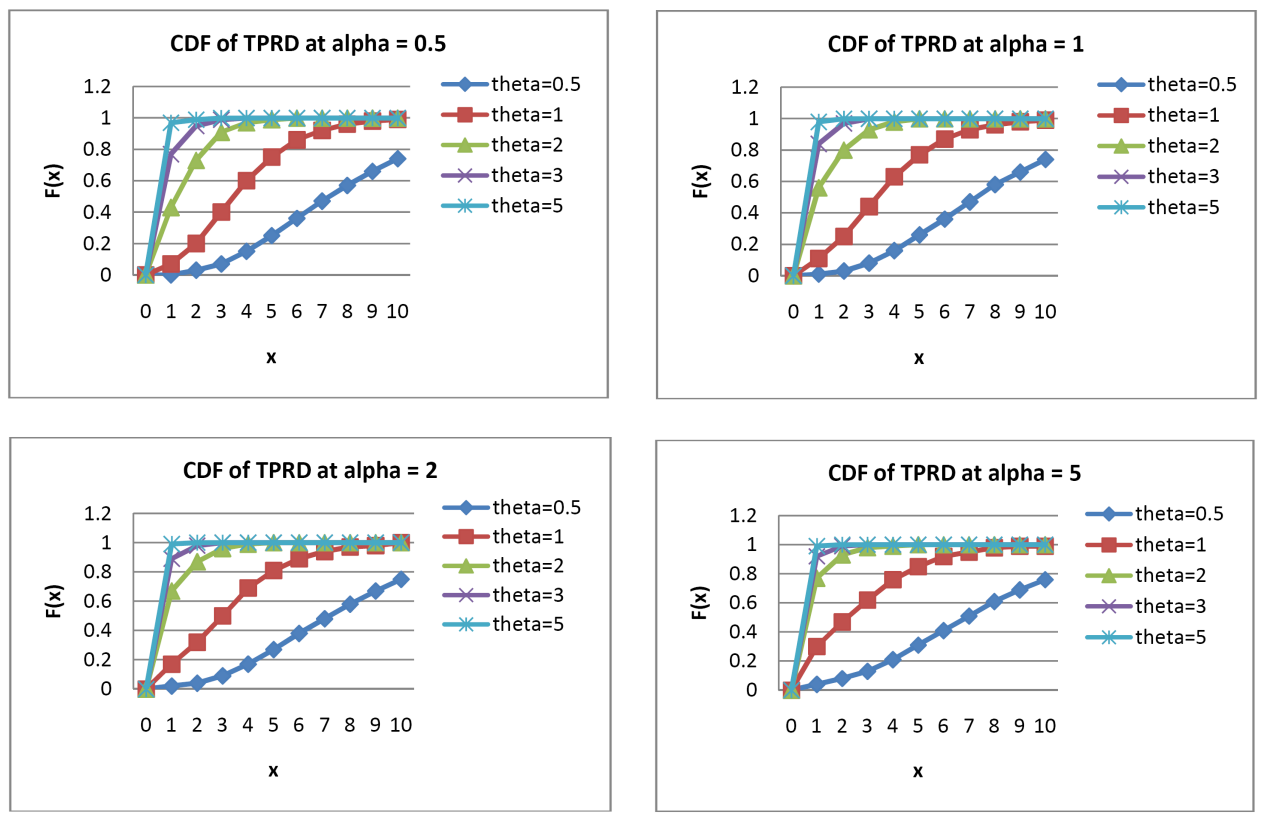

Figure 2. Behaviour of the CDF of TPRD for varying values of the parameters $\theta$ and $\alpha$.

\section{Moments and Related Measures}

The $r$ th moment about origin of the two-parameter Rama distribution is obtained as

$$
\mu_{r}^{\prime}=\frac{r !\left[\alpha \theta^{3}+(r+1)(r+2)(r+3)\right]}{\theta^{r}\left(\alpha \theta^{3}+6\right)} ; \quad r=1,2,3, \ldots .
$$

Thus, the first four moments about origin of the two-parameter Rama distribution are given as

$$
\begin{array}{ll}
\mu_{1}^{\prime}=\frac{\alpha \theta^{3}+24}{\theta\left(\alpha \theta^{3}+6\right)}, & \mu_{2}^{\prime}=\frac{2\left(\alpha \theta^{3}+60\right)}{\theta^{2}\left(\alpha \theta^{3}+6\right)}, \\
\mu_{3}^{\prime}=\frac{6\left(\alpha \theta^{3}+120\right)}{\theta^{3}\left(\alpha \theta^{3}+6\right)}, & \mu_{4}^{\prime}=\frac{24\left(\alpha \theta^{3}+210\right)}{\theta^{4}\left(\alpha \theta^{3}+6\right)} .
\end{array}
$$

The relationship between moments about the mean and moments about origin gives the moments about mean of two-parameter Rama distribution as 


$$
\begin{aligned}
& \mu_{2}=\frac{\left(\alpha^{2} \theta^{6}+84 \alpha \theta^{3}+144\right)}{\theta^{2}\left(\alpha \theta^{3}+6\right)^{2}}, \\
& \mu_{3}=\frac{2\left(\alpha^{3} \theta^{9}+198 \alpha^{2} \theta^{6}+324 \alpha \theta^{3}+864\right)}{\theta^{3}\left(\alpha \theta^{3}+6\right)^{3}}, \\
& \mu_{4}=\frac{9\left(\alpha^{4} \theta^{12}+312 \alpha^{3} \theta^{9}+2304 \alpha^{2} \theta^{6}+10368 \alpha \theta^{3}+10368\right)}{\theta^{4}\left(\alpha \theta^{3}+6\right)^{4}} .
\end{aligned}
$$

The coefficient of variation (C.V), coefficient of skewness $\left(\sqrt{\beta_{1}}\right)$, coefficient of kurtosis $\left(\beta_{2}\right)$, index of dispersion $(\gamma)$ of the two-parameter Rama distribution are given as

$$
\begin{aligned}
& c . v=\frac{\sigma}{\mu_{1}^{\prime}}=\frac{\sqrt{\alpha^{2} \theta^{6}+84 \alpha \theta^{3}+144}}{\alpha \theta^{3}+24}, \\
& \sqrt{\beta_{1}}=\frac{\mu_{3}}{\left(\mu_{2}\right)^{3 / 2}}=\frac{2\left(\alpha^{3} \theta^{9}+198 \alpha^{2} \theta^{6}+324 \alpha \theta^{3}+864\right)}{\left(\alpha^{2} \theta^{6}+84 \alpha \theta^{3}+144\right)^{3 / 2}}, \\
& \beta_{2}=\frac{\mu_{4}}{\mu_{2}}=\frac{9\left(\alpha^{4} \theta^{12}+312 \alpha^{3} \theta^{9}+2304 \alpha^{2} \theta^{6}+10368 \alpha \theta^{3}+10368\right)}{\left(\alpha^{2} \theta^{6}+84 \alpha \theta^{3}+144\right)^{2}}, \\
& \gamma=\frac{\sigma^{2}}{\mu_{1}}=\frac{\alpha^{2} \theta^{6}+84 \alpha \theta^{3}+144}{\theta\left(\alpha \theta^{3}+6\right)\left(\alpha \theta^{3}+24\right)} .
\end{aligned}
$$

It can be easily verified that these statistical constants of the two-parameter Rama distribution reduces to the corresponding statistical constants of Rama distribution at $\alpha=1$.
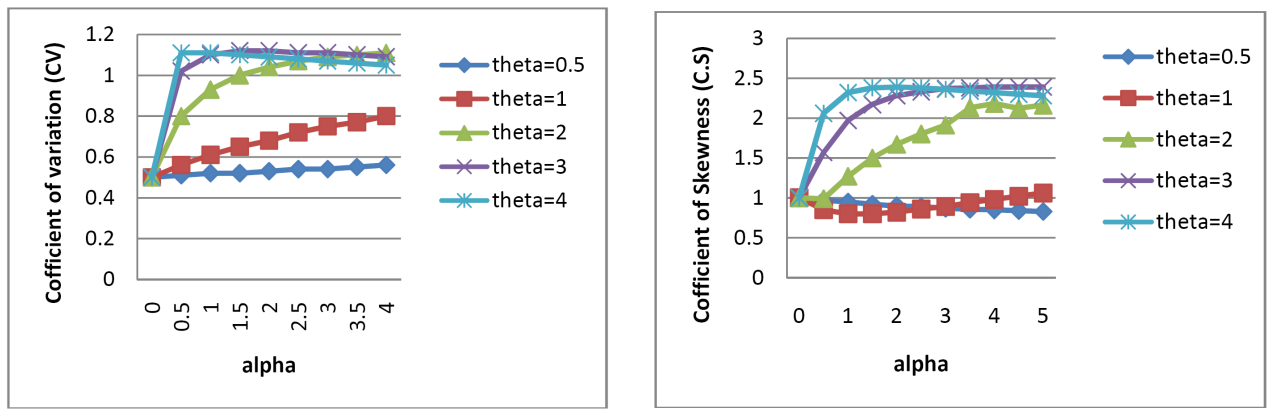

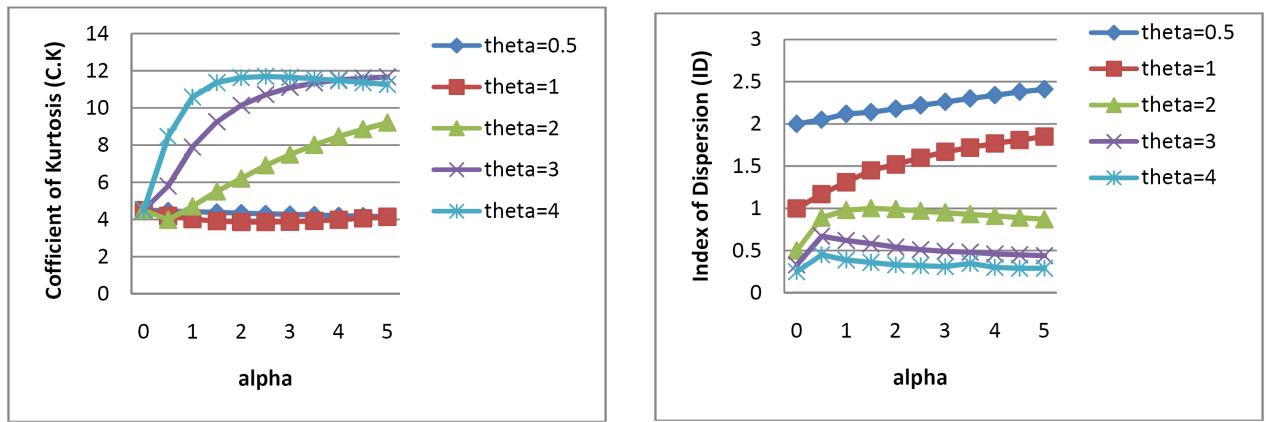

Figure 3. Behaviour of C.V, $\sqrt{\beta_{1}}, \beta_{2}$, and $\gamma$, for varying values of the parameters $\theta$ and $\alpha$.

\section{Hazard Rate and Mean Residual Life Function}

Let $X$ be a continuous random variable with pdf $f(x)$ and cdf $F(x)$. The hazard rate function (also known as failure rate function), $h(x)$ and the mean residual function, $m(x)$ of $x$ are respectively defined as

$$
h(x)=\lim _{\Delta x \rightarrow 0} \frac{p(X<x+\Delta x / X>x)}{\Delta x}=\frac{f(x)}{1-F(x)}
$$

and

$$
m(x)=E[X-x / X>x]=\frac{1}{1-F(x)} \int_{x}^{\infty}[1-F(t)] d t .
$$

The corresponding failure rate function $h(x)$ and the mean residual life function $m(x)$ of the distribution are thus given as

$$
\begin{aligned}
h(x)= & \frac{\theta^{4}\left(\alpha+x^{3}\right)}{\left(\theta^{3} x^{3}+3 \theta^{2} x^{2}+6 \theta x+\alpha \theta^{3}+6\right)}, \\
m(x)= & \frac{1}{\left(\theta^{3} x^{3}+3 \theta^{2} x^{2}+6 \theta x+\alpha \theta^{3}+6\right) e^{-\theta x}} \\
& \times \int_{x}^{\infty}\left(\theta^{3} x^{3}+3 \theta^{2} x^{2}+6 \theta x+\alpha \theta^{3}+6\right) e^{-\theta x}
\end{aligned}
$$




$$
=\frac{\theta^{3} x^{3}+6 \theta^{2} x^{2}+18 \theta x+\alpha \theta^{3}+24}{\theta\left(\theta^{3} x^{3}+3 \theta^{2} x^{2}+6 \theta x+\alpha \theta^{3}+6\right)} \text {. }
$$

It can be verified that $h(0)=\frac{\alpha \theta^{4}}{\alpha \theta^{3}+6}=f(0)$ and $m(0)=\frac{\alpha \theta^{3}+24}{\theta\left(\alpha \theta^{3}+6\right)}=\mu_{1}^{\prime}$.

The expression for $h(x)$ and $m(x)$ of the two-parameter Rama distribution reduces to the corresponding $h(x)$ and $m(x)$ of Rama distribution at $\alpha=1$.

Thus, the graphs of $h(x)$ and $m(x)$ are given below.
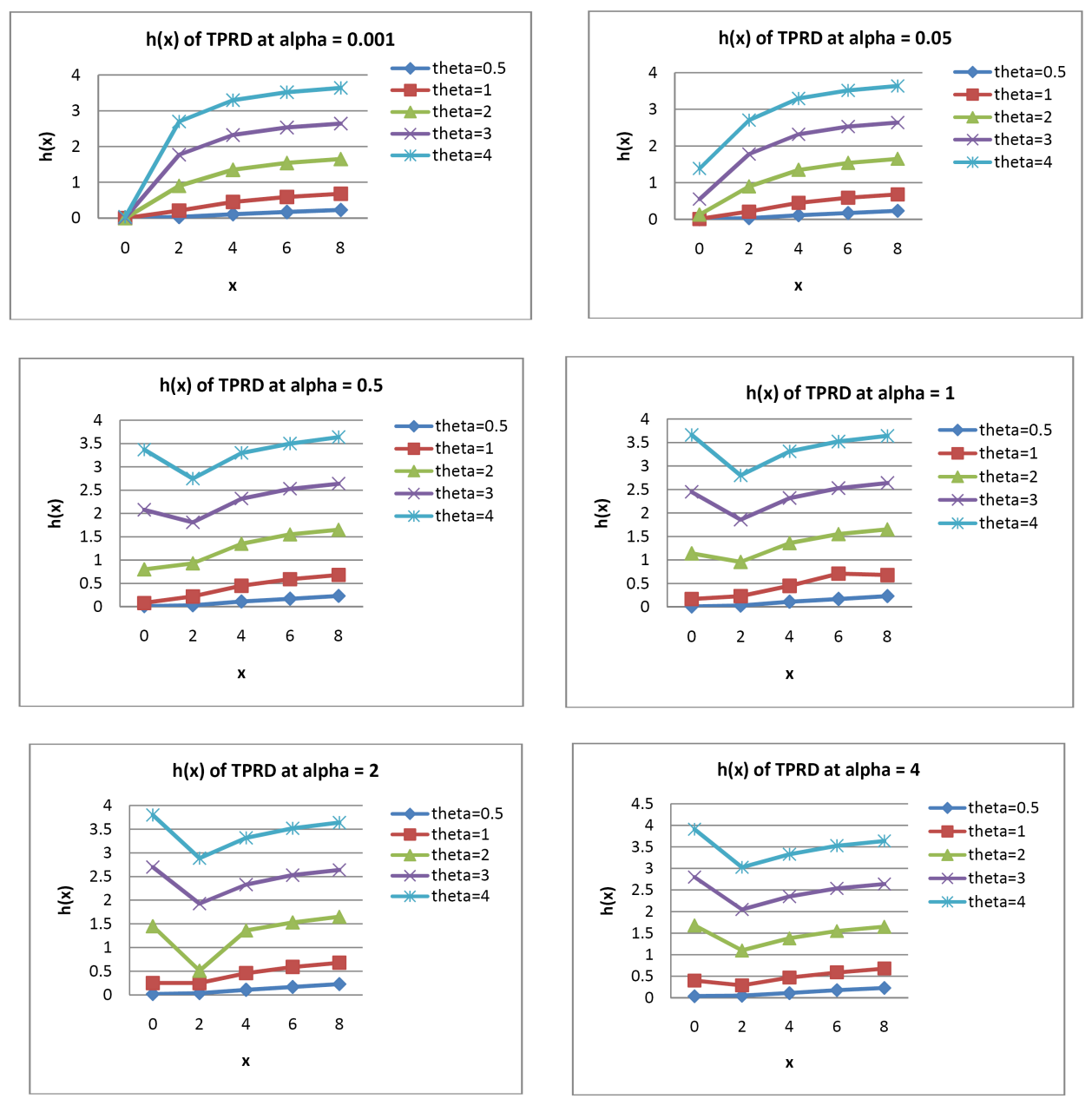

Figure 4. Behaviour of $h(x)$ of TPRD for selected values of parameters $\theta$ and $\alpha$. 

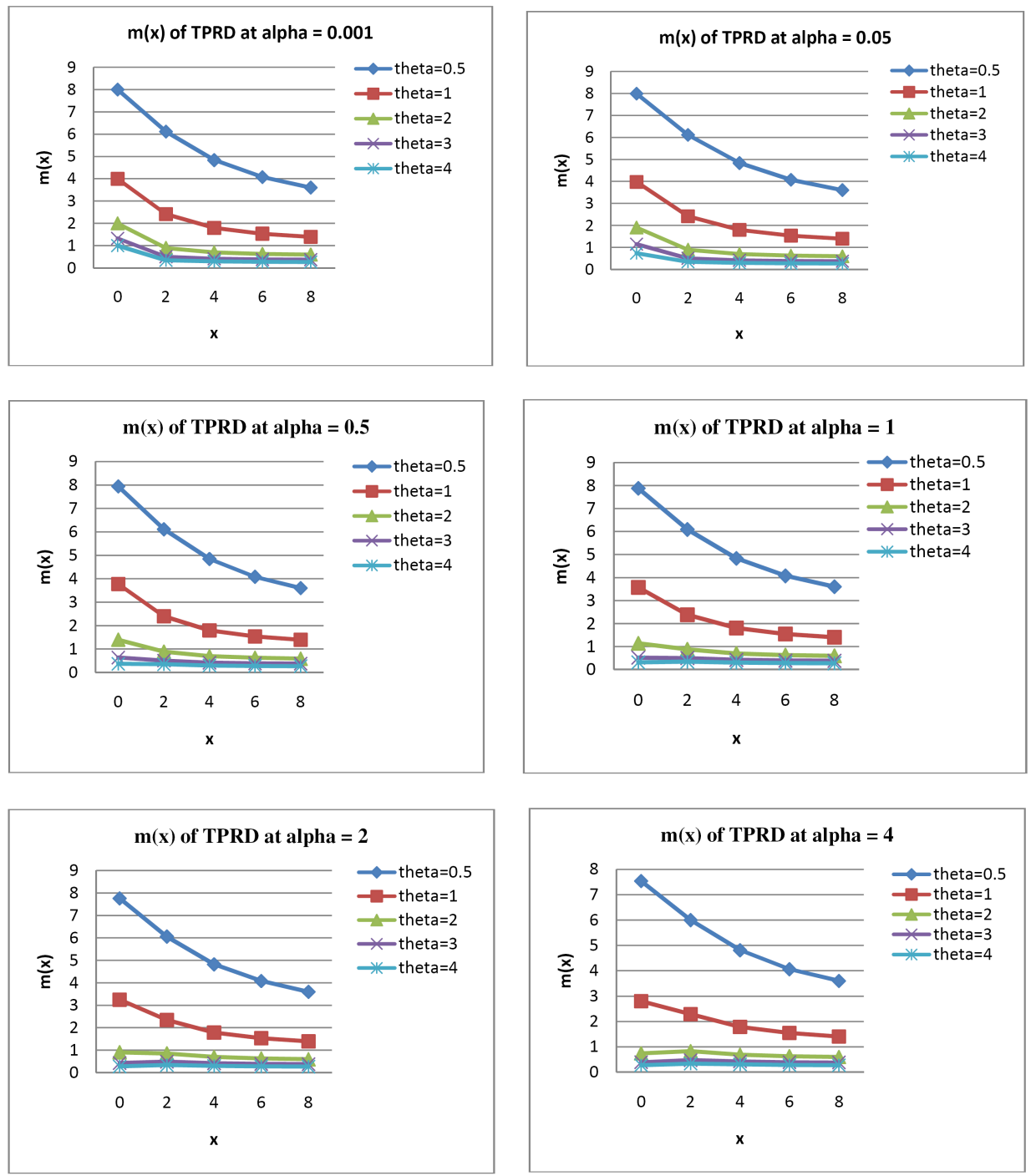

Figure 5. Behaviour of $m(x)$ of TPRD for selected values of parameters $\theta$ and $\alpha$.

\section{Stochastic Orderings}

Stochastic ordering of positive continuous random variables is an important tool for judging the comparative behavior. A random variable $X$ is said to be smaller than a random variable $Y$ in the

(i) Stochastic order $X \leq_{s t} Y$ if $F_{X}(x) \geq F_{Y}(x)$ for all $x$. 
(ii) Hazard rate order $X \leq_{h r} Y$ if $h_{X}(x) \geq h_{Y}(x)$ for all $x$.

(iii) Mean residual life order $X \leq_{m r l} Y$ if $m_{X}(x) \geq m_{Y}(x)$ for all $x$.

(iv) Likelihood ratio order $X \leq_{l r} Y$ if $\frac{f_{X}(x)}{f_{Y}(x)}$ decreases in $x$.

The following results due to Shaked and Shanthikumar [9] are well known for establishing stochastic ordering of distributions.

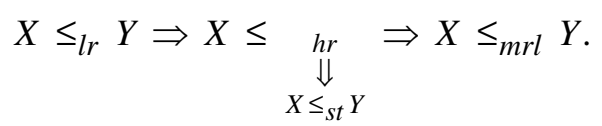

The two-parameter Rama distribution (TPRD) is ordered with respect to the strongest likelihood ratio ordering as shown in the following theorem.

Theorem. Let $X$ be two-parameter Rama distribution $\left(\alpha_{1}, \theta_{1}\right)$ and $Y$ be twoparameter Rama distribution $\left(\alpha_{2}, \theta_{2}\right)$. If $\alpha_{1}=\alpha_{2}$ and $\theta_{1} \geq \theta_{2}$ (or if $\theta_{1}=\theta_{2}$ and $\left.\alpha_{1} \geq \alpha_{2}\right)$, then $X \leq_{l r} Y$ and hence $X \leq_{h r} Y, X \leq_{m r l} Y$ and $X \leq_{s t} Y$.

Proof. We have

$$
\frac{f_{X}\left(x ; \theta_{1}, \alpha_{1}\right)}{f_{Y}\left(x ; \theta_{2}, \alpha_{2}\right)}=\frac{\theta_{1}^{4}\left(\alpha_{2} \theta_{2}^{3}+6\right)}{\theta_{2}^{4}\left(\alpha_{1} \theta_{1}^{3}+6\right)} \frac{\left(\alpha_{1}+x^{3}\right)}{\left(\alpha_{2}+x^{3}\right)} e^{-\left(\theta_{1}-\theta_{2}\right) x} ; x>0 .
$$

Now

$$
\begin{gathered}
\ln \frac{f_{X}\left(x ; \theta_{1}, \alpha_{1}\right)}{f_{Y}\left(x ; \theta_{2}, \alpha_{2}\right)}=\ln \left[\frac{\theta_{1}^{4}\left(\alpha_{2} \theta_{2}^{3}+6\right)}{\theta_{2}^{4}\left(\alpha_{1} \theta_{1}^{3}+6\right)}\right]+\ln \left[\frac{\left(\alpha_{1}+x^{3}\right)}{\left(\alpha_{2}+x^{3}\right)}\right]-\left(\theta_{1}-\theta_{2}\right) x \\
\frac{d}{d x} \ln \frac{f_{X}\left(x ; \theta_{1}, \alpha_{1}\right)}{f_{Y}\left(x ; \theta_{2}, \alpha_{2}\right)}=\frac{3 x^{2}\left(\alpha_{2}-\alpha_{1}\right)}{\left(\alpha_{1}+x^{3}\right)\left(\alpha_{2}+x^{3}\right)}-\left(\theta_{1}-\theta_{2}\right) .
\end{gathered}
$$

Thus, for $\left(\theta_{1}>\theta_{2}\right.$ and $\left.\alpha_{1}=\alpha_{2}\right)$ or $\left(\alpha_{1}>\alpha_{2}\right.$ and $\left.\theta_{1}=\theta_{2}\right)$,

$$
\frac{d}{d x} \ln \frac{f_{X}\left(x ; \theta_{1}, \alpha_{1}\right)}{f_{Y}\left(x ; \theta_{2}, \alpha_{2}\right)}<0
$$

This means that $X \leq_{l r} Y$ and hence $X \leq_{h r} Y, X \leq_{m r l} Y$ and $X \leq_{s t} Y$. This shows the flexibility of the two-parameter Rama distribution over Rama distribution. 


\section{Mean Deviation}

The amount of scatter in a population is evidently measured to some extent by the totality of deviations from the mean and the median. These are known as mean deviation about the mean and mean deviation about the median and are, respectively, defined by

$$
\delta_{1}(x)=\int_{0}^{\infty}|X-\mu| f(x) d x \quad \text { and } \quad \delta_{2}(x)=\int_{0}^{\infty}|X-M| f(x) d x,
$$

where $\mu=E(x)$ and $M=\operatorname{median}(X)$. The measures $\delta_{1}(x)$ and $\delta_{2}(x)$ can be calculated using the following relationships:

$$
\begin{aligned}
\delta_{1}(x) & =\int_{0}^{\mu}(\mu-x) f(x) d x+\int_{\mu}^{\infty}(x-\mu) f(x) d x \\
& =\mu F(\mu)-\int_{0}^{\mu} x f(x) d x-\mu[1-F(\mu)]+\int_{\mu}^{\infty} x f(x) d x \\
& =2 \mu F(\mu)-2 \mu+2 \int_{\mu}^{\infty} x f(x) d x \\
& =2 \mu F(\mu)-2 \int_{0}^{\mu} x f(x) d x
\end{aligned}
$$

and

$$
\begin{aligned}
\delta_{2}(x) & =\int_{0}^{M}(M-x) f(x) d x+\int_{M}^{\infty}(M-\mu) f(x) d x \\
& =M F(M)-\int_{0}^{M} x f(x) d x-M[1-F(M)]+\int_{M}^{\infty} x f(x) d x \\
& =\mu+2 \int_{M}^{\infty} x f(x) d x \\
& =\mu-2 \int_{0}^{M} x f(x) d x .
\end{aligned}
$$

Using pdf (2.1) and the mean of two-parameter Rama distribution, it can be written as; 
$\int_{0}^{\mu} x f(x) d x=\mu-\frac{\left[\theta^{4}\left(\mu^{4}+\alpha \mu\right)+4 \theta^{3} \mu^{3}+\alpha \theta^{3}+12 \theta^{2} \mu^{2}+24 \theta \mu+24\right]}{\theta\left(\alpha \theta^{3}+6\right)} e^{-\theta \mu}$,

$$
\int_{0}^{M} x f(x) d x=\mu-\frac{\left[\theta^{4}\left(M^{4}+\alpha M\right)+\alpha \theta^{3}+4 \theta^{3} M^{3}+12 \theta^{2} M^{2}+24 \theta M+24\right]}{\theta\left(\alpha \theta^{4}+6\right)} e^{-\theta x} .
$$

Using expression (6.1), (6.2), (6.3) and (6.4), the mean deviation about the mean $\delta_{1}(x)$ and the mean deviation about the median $\delta_{2}(x)$ of the two-parameter Rama distribution are expressed as

$$
\begin{aligned}
& \delta_{1}(x)=\frac{2\left(\alpha \theta^{3}+\theta^{3} \mu^{3}+6 \theta^{2} \mu^{2}+18 \theta \mu+24\right) e^{-\theta \mu}}{\theta\left(\alpha \theta^{3}+6\right)} \\
& \delta_{2}(x)=\frac{2\left[\theta^{4}\left(M^{4}+\alpha M\right)+\alpha \theta^{3}+4 \theta^{3} M^{3}+12 \theta^{2} M^{2}+24 \theta M+24\right) e^{-\theta M}}{\theta\left(\alpha \theta^{3}+6\right)}-\mu .
\end{aligned}
$$

\section{Bonferroni and Lorenz Curves}

The Bonferroni and Lorenz curves introduced by Bonferroni [10] and Bonferroni and Gini indices have applications in almost every fields of knowledge including economics to study income and poverty of any state. Its relevance is also in other fields like reliability, demography, insurance and medicine. The Bonferroni and Lorenz curves are defined as

$$
\begin{aligned}
B(p) & =\frac{1}{p \mu} \int_{0}^{q} x f(x) d x \\
& =\frac{1}{p \mu}\left[\int_{0}^{\infty} x f(x) d x-\int_{q}^{\infty} x f(x) d x\right] \\
& =\frac{1}{p \mu}\left[\mu-\int_{q}^{\infty} x f(x) d x\right]
\end{aligned}
$$

and

$$
L(p)=\frac{1}{\mu} \int_{0}^{q} x f(x) d x
$$




$$
\begin{aligned}
& =\frac{1}{\mu}\left[\int_{0}^{\infty} x f(x) d x-\int_{q}^{\infty} x f(x) d x\right] \\
& =\frac{1}{\mu}\left[\mu-\int_{q}^{\infty} x f(x) d x\right]
\end{aligned}
$$

or equivalently

$$
B(p)=\frac{1}{p \mu} \int_{0}^{p} F^{-1}(x) d x
$$

and

$$
L(p)=\frac{1}{\mu} \int_{0}^{p} F^{-1}(x) d x
$$

where $\mu=E(x)$ and $q=F^{-1}(p)$.

The Bonferroni and Gini indices are thus defined as

$$
\begin{gathered}
B=1-\int_{0}^{1} B(p) d p, \\
G=1-2 \int_{0}^{1} L(p) d p,
\end{gathered}
$$

respectively.

Using the pdf of two-parameter Rama distribution (2.1), we obtain

$$
\int_{q}^{\infty} x f(x) d x=\frac{\left\{\alpha \theta^{4} q+\theta^{4}\left(q^{4}+\alpha\right)+4 \theta^{3} q^{3}+12 \theta^{2} q^{2}+24 \theta q+24\right\} e^{-\theta q}}{\theta\left(\alpha \theta^{3}+6\right)} .
$$

Now using equation (7.7) in (7.1) and (7.2)

$$
\begin{aligned}
& B(p)=\frac{1}{p}\left[1-\frac{\left\{\alpha \theta^{3}+\theta^{4}\left(q^{4}+q\right)+4 \theta^{3} q^{3}+12 \theta^{2} q^{2}+24 \theta q+24\right\} e^{-\theta q}}{\alpha \theta^{3}+24}\right] \\
& L(p)=1-\frac{\left\{\alpha \theta^{3} q+\theta^{4}\left(q^{4}+q\right)+4 \theta^{3} q^{3}+12 \theta^{2} q^{2}+24 \theta q+24\right\} e^{-\theta q}}{\alpha \theta^{3}+24}
\end{aligned}
$$


Now using equations (7.8) and (7.9), the Bonferroni and Gini indices of the TPRD are thus obtained as

$$
\begin{gathered}
B=1-\frac{\left\{\theta^{4} q^{4}+\theta^{4} q+\alpha \theta^{3}+4 \theta^{3} q^{3}+12 \theta^{2} q^{2}+24 \theta q+24\right\} e^{-\theta q}}{\left(\alpha \theta^{3}+24\right)}, \\
G=\frac{2\left\{\alpha \theta^{3} q+\theta^{4}\left(q^{4}+q\right)+4 \theta^{3} q^{3}+12 \theta^{2} q^{2}+24 \theta q+24\right\} e^{-\theta q}}{\left(\alpha \theta^{3}+24\right)}-1 .
\end{gathered}
$$

\section{Parameter Estimation}

In this section, the estimations of parameters of TPRD using method of moments and method of maximum likelihood were discussed.

\section{Method of Moment Estimates (MOME)}

Since TPRD (2.1) has two parameters to be estimated, the first two moments about the origin are required to estimate the parameters using method of moments. By equating the populations mean to the sample mean, we have

$$
\begin{gathered}
\frac{\mu_{2}^{\prime}}{\left(\mu_{1}^{\prime}\right)}=k, \\
\frac{2\left(\theta^{3}+60\right)}{\theta^{2}\left(\theta^{3}+6\right)} \frac{\left(\alpha \theta^{3}+24\right)}{\theta\left(\alpha \theta^{3}+6\right)}=k, \\
\frac{2\left(\theta^{3}+60\right)}{\theta^{2}\left(\theta^{3}+6\right)} \times \frac{\theta^{2}\left(\alpha \theta^{3}+6\right)^{2}}{\left(\alpha \theta^{3}+24\right)^{2}} k, \\
\frac{2\left(\theta^{3}+60\right)\left(\alpha \theta^{3}+6\right)}{\left(\alpha \theta^{3}+24\right)^{2}}=k .
\end{gathered}
$$

Taking $\theta^{3}=\beta$, we obtain a quadratic equation

$$
\beta^{2}(2-k)+4 \beta(33-12 k)+12(60-48 k)=0 .
$$


It should be noted that for real value of $\beta, k \leq 2.083$. By replacing $\mu_{1}^{\prime}$ and $\mu_{2}^{\prime}$ by their respective sample moments in (8.1), an estimate of $k$ can be obtained and substituting the value of $k$ in equation (8.2), value of $\beta$ can be obtained. Also taking $\alpha \theta^{3}=\beta$ in the expression for the mean of TPRD, we obtain the method of moment estimate (MOME) $\hat{\theta}$ of $\theta$ as

$$
\hat{\theta}=\frac{\beta+24}{(\beta+6) \bar{x}} \text { and } \hat{\alpha}=\frac{\beta}{\hat{\theta}^{3}}=\frac{\beta(\beta+6)^{3} \bar{x}^{3}}{(\beta+24)^{3}} \text {. }
$$

\section{Method of Maximum Likelihood Estimates}

Let $\left(x_{1}, x_{2}, x_{3}, \ldots, x_{n}\right)$ be a random sample of size $n$ from (2.1). The likelihood function, $L$ of two-parameter Rama distribution is given by

$$
L=\left(\frac{\theta^{4}}{\alpha \theta^{3}+6}\right)^{n} \prod_{i=1}^{n}\left(\alpha+x_{i}^{3}\right) e^{-n \theta \bar{X}},
$$

where $\bar{X}$ is the sample mean.

The natural log likelihood function is thus obtained as

$$
\ln L=n \ln \left(\frac{\theta^{4}}{\alpha \theta^{3}+6}\right)+\sum_{i=1}^{n} \ln \left(\alpha+x_{i}^{3}\right)-n \theta \bar{X} .
$$

The maximum likelihood estimates, $\hat{\theta}$ of $\theta$ and $\hat{\alpha}$ of $\alpha$ is the solution of the equation $\frac{\partial \ln L}{\partial \theta}=0$ and $\frac{\partial \ln L}{\partial \alpha}=0$ and it can be obtained by solving the following non-linear equations

$$
\frac{\partial \ln L}{\partial \theta}=\frac{4 n}{\theta}-\frac{3 \alpha n \theta^{2}}{\alpha \theta^{3}+6}-n \bar{x}=0,
$$

where $\bar{x}$ is the sample mean

$$
\frac{\partial \ln L}{\partial \alpha}=\frac{-n \theta^{3}}{\left(\alpha \theta^{3}+6\right)}+\sum_{i=1}^{n} \frac{1}{\left(\alpha+x_{i}^{3}\right)}=0 .
$$


These two natural log likelihoods cannot be solved directly because they cannot be expressed in closed forms. The MLE's of $\theta$ and $\alpha$ can be computed directly by solving the natural log likelihood equations using Newton-Raphson iteration method using Rsoftware till sufficiently close values of $\hat{\theta}$ and $\hat{\alpha}$ are obtained.

\section{Data Analysis}

In this section the application of TPRD with a real life data set using maximum likelihood estimate has been discussed. The data set was collected from a general hospital in eastern Nigeria on 200 patients who were diagnosed with hepatitis B where second visit is the event of interest.

$\begin{array}{rrrrrrrrrrrr}27 & 32 & 8 & 30 & 34 & 23 & 41 & 36 & 28 & 16 & 30 & 3 \\ 24 & 7 & 7 & 30 & 33 & 17 & 38 & 36 & 29 & 17 & 14 & 7 \\ 27 & 12 & 32 & 32 & 26 & 15 & 31 & 34 & 28 & 27 & 6 & 7 \\ 28 & 44 & 31 & 27 & 32 & 7 & 32 & 35 & 26 & 16 & 3 & 8 \\ 28 & 35 & 32 & 29 & 28 & 27 & 32 & 33 & 10 & 14 & 10 & 1 \\ 26 & 23 & 32 & 29 & 27 & 31 & 32 & 36 & 28 & 39 & 10 & 2 \\ 26 & 23 & 31 & 29 & 30 & 31 & 28 & 34 & 23 & 39 & 10 & 9 \\ 26 & 18 & 31 & 46 & 30 & 39 & 33 & 34 & 31 & 19 & 3 & 6 \\ 27 & 23 & 30 & 46 & 38 & 39 & 33 & 37 & 30 & 29 & 3 & 6 \\ 32 & 26 & 30 & 33 & 48 & 40 & 32 & 30 & 25 & 30 & 3 & 7\end{array}$

The goodness of fit of TPRD was compared with Rama, 2-parameter Akash, Ishita, Akash, Shanker, Lindley, and Exponential distributions.

In order to compare these distributions, values of $-2 \ln L$, AIC (Akaike Information Criterion), BIC (Bayesian Information Criterion) for the real data sets have been computed. The formulae for computing AIC and BIC are as follows:

$$
A I C=-2 \ln L+2 k, \quad B I C=-2 \ln L+k \ln n,
$$

where $k$ is the number of parameters and $n$ is the sample size. 
Table 1. MLE's, $-2 \ln L$, AIC and BIC of the fitted distribution of the given data set.

\begin{tabular}{|cccccc|}
\hline \multirow{2}{*}{ Distribution } & \multicolumn{2}{c}{ MLE Estimates } & & & \\
\cline { 2 - 5 } & $\hat{\theta}$ & $\hat{\alpha}$ & $-2 \ln L$ & AIC & BIC \\
\hline TPRD & 0.14428 & 266.6997 & 942.328 & 946.328 & 951.9032 \\
\hline TPAD & 0.11214 & 14.35639 & 953.251 & 957.251 & 962.8263 \\
\hline Akash & 0.11815 & & 958.548 & 960.548 & 963.334 \\
\hline Ishita & 0.11868 & & 960.290 & 962.290 & 965.078 \\
\hline Rama & 0.15818 & & 967.557 & 969.557 & 972.344 \\
\hline Shanker & 0.07886 & & 967.613 & 969.613 & 972.401 \\
\hline Lindley & 0.07632 & & 969.569 & 971.569 & 974.356 \\
\hline Exponential & 0.03956 & & 1015.156 & 1017.156 & 1019.943 \\
\hline
\end{tabular}

It is obvious from Table 1 that TPRD gives much better fit than other distributions mentioned above; hence the TPRD can be considered as an important two-parameter lifetime distribution.

\section{Conclusions}

A two parameter Rama distribution (TPRD) has been proposed which has its base from Rama distribution. Its mathematical and statistical properties which include moments, coefficient of variation, skewness, kurtosis, index of dispersion, hazard rate function, mean residual life function and stochastic ordering have been discussed. Further, expressions for Bonferroni and Lorenz curves of the proposed distribution have been derived. The method of moments and the method of maximum likelihood estimation have also been discussed for estimating its parameter. An example of real life data set has been presented to show the application and goodness of fit of the two parameter Rama distribution over Rama, 2-parameter Akash, 2-parameter Lindley, Ishita, Akash, Shanker, Lindley, and Exponential distributions. The values of the MLE's, $-2 \ln L$, AIC and BIC of the distributions shows the superiority of the (TPRD) over the other distributions. Hence (TPRD) can be seen as an important distribution in modeling life time data. 


\section{References}

[1] R. Shanker, Rama distribution and its application, International Journal of Statistics and Application 7(1) (2017), 26-35.

[2] D. V. Lindley, Fiducial distributions and Bayes' theorem, J. Roy. Statist. Soc. Ser. B 20(1) (1958), 102-107. https://doi.org/10.1111/j.2517-6161.1958.tb00278.x

[3] R. Shanker, Akash distribution and its applications, International Journal of Probability and Statistics 4(3) (2015a), 65-75

[4] R. Shanker, Shanker distribution and its application, International Journal of Statistics and Application 5(6) (2015b), 338-348.

[5] R. Shanker, Amarendra distribution and its applications, American Journal of Mathematics and Statistics 6(1) (2016a), 44-56.

[6] R. Shanker, Aradhana distribution and its applications, International Journal of Statistics and Application 6(1) (2016b), 23-34.

[7] M. E. Ghitany, B. Atieh and S. Nadarajah, Lindley distribution and its application, Math. Comput. Simulation 78(4) (2008), 493-506.

https://doi.org/10.1016/j.matcom.2007.06.007

[8] R. Shanker and K. K. Shukla, On two-parameter Akash distribution, Biom. Biostat. Int. J. 6(5) (2017), 00178. https://doi.org/10.15406/bbij.2017.06.00178

[9] M. Shaked and J. G. Shanthikumar, Stochastic Orders and their Applications, New York, USA: Academic Press, 1994.

[10] C. E. Bonferroni, Elementi di Statistica Generale, Seeber, Firenze, 1930. 